\title{
OPERATOR-VALUED FREE ENTROPY AND MODULAR FRAMES*
}

\author{
MAOZHENG $\mathrm{GUO}^{\dagger}$, BIN MENG ${ }^{\dagger}$, AND XIAOHONG $\mathrm{CAO}^{\dagger}$ \\ Dedicated to George Papanicolaou on the occasion of his 60th birthday
}

\begin{abstract}
We introduce the operator-valued relative free entropy $\chi_{\mathcal{B}}^{*}\left(X_{1}, X_{2}, \cdots, X_{n}: \mathcal{B}\right)$ of a family of self-adjoint random variables $X_{1}, X_{2}, \cdots, X_{n}$ in a $\mathcal{B}$-valued noncommutative probability space $\left(\mathcal{A}, E_{\mathcal{B}}, \mathcal{B}\right)$. This notion extends $D$. Voiculescu's relative free entropy $\Phi^{*}$ which defined in a tracial $\mathrm{W}^{*}$-noncommutative probability space to a more general context. The free entropy of a semicircular variable with conditional expectation covariance can be computed by using the modular frames and then we point out the relation between the free entropy of a semicircular variable and the index of a conditional expectation. At last, we obtain an estimate of the free entropy dimension $\delta_{\mathcal{B}, \tau}^{*}$.
\end{abstract}

Key words. operator-valued free entropy; modular frame; free Fisher information; index

AMS subject classifications. Primary 46L54, 42C15; Secondary 46L08; 46L09

1. Introduction. About twenty years ago, Dan Voiculescu introduced a noncommutative probability (or "free probability") theory in the framework of operator algebras. The theory soon became a powerful tool in the study of operator algebras. Especially, its connection with random matrices and the notion of free entropy are very important in the study of von Neumann algebras. Dan Voiculescu provided two versions of free entropy: one is defined by matricial microstates approach and the other is defined by infinitesimal microstates approach. Using the first version of free entropy, Voiculescu proved the absence of Cartan subalgebras in free group factors (see [15]) and Ge found the primeness of free group factors (see [6]). However, for a number of questions, matricial microstates pose many technical difficulties and the proofs of some important desired properties of the free entropy have remained out of reach, so Voiculescu provided another route to free entropy- infinitesimal microstates approach, which avoids matricial microstates. In this new approach the free fisher information measure $\Phi^{*}$ comes first and the free entropy is derived from it ([16],[17]).

In this paper we introduce the relative free entropy in the operator-valued framework following D. Voiculescu's idea. Originally, Voiculescu defined the relative free entropy $\chi^{*}$ in a tracial $\mathrm{W}^{*}$ algebras $\mathcal{A}$ (that is, there is a faithful normal tracial state $\tau$ on $\mathcal{A}$ ). Now it is defined in an operator-valued noncommutative probability space and $\tau$ is replaced by a conditional expectation $E_{\mathcal{B}}$ of $\mathcal{A}$ onto a subalgebra $\mathcal{B}$. It is defined in a way that is almost identical to the definition of $\chi^{*}$. So in order to define relative free entropy in an operator-valued noncommutative probability space we must define the operator-valued free Fisher information first. In fact We have introduced the notion and investigated some properties of operator-valued free Fisher information $\Phi_{\mathcal{B}}^{*}$ in [7]. These properties are very similar to those of $\Phi^{*}$ but we can not follow D. Voiculescu's method to prove them since $E_{\mathcal{B}}$ is not tracial in general. The main tools we used was R. Speicher's cumulant function theory for noncommutative variables. In this paper we review some of them and since the free entropy $\chi_{\mathcal{B}}^{*}$ is defined by free Fisher information, $\chi_{\mathcal{B}}^{*}$ has the corresponding properties. A main result in this paper is that the free entropy of a $E$-semicircular variable is very closely related to

\footnotetext{
* Received July 8, 2004; accepted for publication January 15, 2005.

${ }^{\dagger}$ LMAM, School of Mathematical Science, Peking University, Beijing, 100871, P. R. China (maguo@pku.edu.cn).
} 
the index of $E$. In fact we obtain an explicit formula of $\chi_{\mathcal{B}}^{*}(X: \mathcal{B})$ where $X$ is a $E$-semicircular variable. At last, we obtain an estimate of the relative free entropy dimension $\delta_{\mathcal{B}, \tau}^{*}$. The estimate of free entropy dimension is very important since it is essential in D. Voiculescu's and Ge's famous works in [15],[6].

2. Operator-valued free Fisher information. Let $\mathcal{A}$ be a von Neumann algebra and let $\mathcal{B}$ be a von Neumann subalgebra of $\mathcal{A}$. $E_{\mathcal{B}}$ is a normal conditional expectation of $\mathcal{A}$ onto $\mathcal{B}$. Then $\left(\mathcal{A}, E_{\mathcal{B}}, \mathcal{B}\right)$ is called an operator-valued $W^{*}$-noncommutative probability space and an element in $\mathcal{A}$ will be called a random variable. In this paper we always suppose the conditional expectations are faithful normal. We refer the readers to [18] for more details on the operator-valued noncommutative probability and we only restate the definition of amalgamated freeness here.

Definition 2.1. [18] Let $\left(\mathcal{A}, E_{\mathcal{B}}, \mathcal{B}\right)$ be a noncommutative probability space, and let $\mathcal{B} \subset \mathcal{A}_{i} \subset \mathcal{A},(i \in I)$ be subalgebras. The family $\left(\mathcal{A}_{i}\right)_{i \in I}$ will be called $\mathcal{B}$-free (or free with amalgamation over $\mathcal{B})$ if

$$
E_{\mathcal{B}}\left(a_{1} a_{2} \cdots a_{n}\right)=0
$$

whenever $a_{j} \in \mathcal{A}_{i_{j}}$ with $i_{1} \neq i_{2} \neq \cdots \neq i_{n}$ and $E_{\mathcal{B}}\left(a_{j}\right)=0,1 \leq j \leq n$. A family of random variables $a_{i} \in \mathcal{A},(i \in I)$ will be called $\mathcal{B}$-free if the family of subalgebras generated by $\left(\mathcal{B} \cup\left\{a_{i}\right\}\right)_{i \in I}$ is $\mathcal{B}$-free.

REMARK 2.2. Sometimes we can choose $E_{\mathcal{B}}$ in a nature way. For instance, let $\tau$ be a faithful normal state on $\mathcal{A}$ and satisfy $\sigma_{t}^{\tau}(\mathcal{B}) \subseteq \mathcal{B}, \forall t \in \mathbb{R}$, where $\sigma_{t}^{\tau}$ is the modular operator. Then from [13], there exists a $\tau$-compatible conditional expectation $E_{\mathcal{B}}$ of $\mathcal{A}$ onto $\mathcal{B}$. Obviously, $E_{\mathcal{B}}$ is faithful normal.

Obviously, we can define the $\mathcal{B}$-valued inner product on $\mathcal{A}$ by $\langle x, y\rangle_{\mathcal{B}}=E_{\mathcal{B}}\left(x^{*} y\right)$ and its Hilbert $\mathrm{C}^{*}$-module completion will be denoted by $L_{\mathcal{B}}^{2}(\mathcal{A})$.

The classical Fisher information is derived from the statistial estimation theory which introduced by R. A. Fisher. If $f$ is a bounded real random variable and the distribution of $f$ is Lebesgue absolutely continuous with density $p$ then the Fisher information $\Phi(f)$ is defined by

$$
\Phi(f)=\int \frac{\left(p^{\prime}(t)\right)^{2}}{p(t)} d t
$$

Another way to get the formular for $\Phi$ is to consider the derivation $\frac{d}{d t}$ on $L^{2}(\mathbb{R}, p d x)$. If 1 is in the domain of $\left(\frac{d}{d t}\right)^{*}$ then

$$
\Phi(f)=\left\|\left(\frac{d}{d t}\right)^{*} 1\right\|^{2}
$$

We refer the readers to [3] for more details on Fisher information.

By analogy with the classical case D. Voiculescu introduced the free Fisher information of a family of self-adjoint random variables in a tracial $\mathrm{W}^{*}$ noncommutative probability space $[16,17]$. In $[7,8]$ we considered the free Fisher information in an operator-valued probability spaces and followed D. Voiculescu's idea we introduced the conjugate variable of a self-adjoint random variable first. Now we review some definitions and propositions on the operator-valued free Fisher information. 
Definition 2.3. [8] Let $\left(\mathcal{A}, E_{\mathcal{B}}, \mathcal{B}\right)$ be a $\mathcal{B}$-valued noncommutative probability space. Suppose $\mathcal{B} \subseteq \mathcal{C} \subseteq \mathcal{A}$ is a von Neumann subalgebra and $X=X^{*} \in \mathcal{A}$ is algebraically free from $\mathcal{C}$ modulo $\mathcal{B}$. If there exists $\xi \in L_{\mathcal{B}}^{2}(\mathcal{C}[X])$ satisfying

$$
E_{\mathcal{B}}\left(\xi c_{0} X \cdots X c_{n}\right)=\sum_{j=1}^{n} E_{\mathcal{B}}\left(c_{0} X \cdots X c_{j-1}\right) E_{\mathcal{B}}\left(c_{j} X \cdots X c_{n}\right),
$$

then $\xi$ will be called the conjugate variable of $X$ w.r.t. $\mathcal{C}$ and denoted by $\mathcal{J}_{\mathcal{B}}(X: \mathcal{C})$, where $L_{\mathcal{B}}^{2}(\mathcal{C}[X])$ is the Hilbert $\mathcal{B}$-module generated by $\mathcal{C}[X]$ whose inner product induced by $E_{\mathcal{B}}$. The free Fisher information $\Phi_{\mathcal{B}}^{*}(X: \mathcal{C})$ is defined by

$$
\Phi_{\mathcal{B}}^{*}(X: \mathcal{C})=E_{\mathcal{B}}\left(\mathcal{J}_{\mathcal{B}}(X: \mathcal{C}) \mathcal{J}_{\mathcal{B}}(X: \mathcal{C})^{*}\right)
$$

and the free Fisher information of a sequence of self-adjoint variables $X_{1}, \cdots, X_{n} \in \mathcal{A}$ is defined by

$$
\Phi_{\mathcal{B}}^{*}\left(X_{1}, \cdots, X_{n}: \mathcal{C}\right)=\sum_{i=1}^{n} \Phi_{\mathcal{B}}^{*}\left(X_{i}: \mathcal{C}\left[X_{1}, \cdots, \widehat{X}_{i}, \cdots, X_{n}\right]\right)
$$

where $\mathcal{C}\left[X_{1}, \cdots, \widehat{X}_{i}, \cdots, X_{n}\right]:=\mathcal{C}\left[X_{1}, \cdots, X_{i-1}, X_{i+1}, \cdots, X_{n}\right]$.

From the definition of conjugate variable, it is easy to see $\mathcal{J}_{\mathcal{B}}(X: \mathcal{C})=\left(\partial^{*}(1 \otimes 1)\right)^{*}$, where $\partial$ is the derivative operator:

$$
\begin{aligned}
& \partial: L_{\mathcal{B}}^{2}(\mathcal{C}[X]) \longrightarrow L_{\mathcal{B}}^{2}(\mathcal{C}[X]) \otimes L_{\mathcal{B}}^{2}(\mathcal{C}[X]) \\
& \partial\left(c_{0} X \cdots X c_{n}\right)=\sum_{j=1}^{n}\left(c_{0} X \cdots X c_{j-1}\right) \otimes\left(c_{j} X \cdots X c_{n}\right)
\end{aligned}
$$

and $\partial^{*}$ is its conjugate operator. Thus this definition is an analogue of the classical Fisher information.

Note that if $\mathcal{J}_{\mathcal{B}}(X: \mathcal{C})$ exists then it is unique since $E_{\mathcal{B}}$ is faithful.

We have investigated some properties of operator-valued free Fisher information in our paper [7], which are similar to those of D. Voiculescu's free Fisher information. We proved them mainly by R. Speicher's cummulant function (see $[9,12])$. Here we only list some of them as follows:

Proposition 2.4. [7] Let $X \in \mathcal{A}$ be self-adjoint. We have:

(1) $\mathcal{J}_{\mathcal{B}}(X: \mathcal{C})$ is unique;

$(2) E_{\mathcal{B}}(\mathcal{J}(X: \mathcal{C}) e)=E_{\mathcal{B}}\left(e \mathcal{J}(X: \mathcal{C})^{*}\right), \forall e \in L_{\mathcal{B}}^{2}(\mathcal{C}[X])$.

Lemma 2.5. [7] Let $\left(\mathcal{A}, E_{\mathcal{B}}, \mathcal{B}\right)$ be a $\mathcal{B}$-valued noncommutative probability space and let $E_{\mathcal{C}}$ be a conditional expectation of $\mathcal{A}$ onto $\mathcal{C}$ such that $E_{\mathcal{B}}=E_{\mathcal{B}} E_{\mathcal{C}} . \xi \in \mathcal{A}$ is the conjugate variable of $X$ with respect to $\mathcal{C}$ if and only if $\xi \in L_{\mathcal{B}}^{2}(\mathcal{C}[X])$ and satisfies the following equations:

$$
\begin{cases}k_{\mathcal{C}}^{(1)}(\xi b)=0, & \forall b \in \mathcal{C} ; \\ k_{\mathcal{C}}^{(2)}(\xi \otimes b a)=\delta_{a X} E_{\mathcal{D}}(b), & \forall b \in \mathcal{C}, a \in\{X\} \cup \mathcal{C} ; \\ k_{\mathcal{C}}^{(m+1)}\left(\xi \otimes b_{1} a_{1} \otimes \cdots \otimes b_{m} a_{m}\right)=0, & \forall b_{1}, \cdots, b_{m} \in \mathcal{C} ; m \geq 2,\end{cases}
$$

where $a_{1}, \cdots, a_{m} \in\{X\} \cup \mathcal{C}$, and $k_{\mathcal{C}}$ is the cumulant function induced by $E_{\mathcal{C}}$. 
Proposition 2.6. [7] Let $\mathcal{B}, \mathcal{C}$ be von Neumann subalgebras of $\mathcal{A}$ satisfying $\mathcal{B} \subseteq \mathcal{C}$ and let $X=X^{*} \in \mathcal{A}$ be self-adjoint. Assuming $\mathcal{B}[X]$ and $\mathcal{C}$ are $\mathcal{B}$-free in $\left(\mathcal{A}, E_{\mathcal{B}}, \mathcal{B}\right)$, then $\mathcal{J}_{\mathcal{B}}(X: \mathcal{B})=\mathcal{J}_{\mathcal{B}}(X: \mathcal{C})$. More generally, $\mathcal{J}_{\mathcal{B}}(X: \mathcal{D} \vee \mathcal{C})=\mathcal{J}_{\mathcal{B}}(X: \mathcal{C})$, if $\mathcal{D} \supseteq \mathcal{B}$ are free from $\mathcal{B}[X]$ with amalgamation over $\mathcal{B}$.

Corollary 2.7. [7] Let $X, Y \in \mathcal{A}$ be self-adjoint. Assume $\mathcal{B}[X]$ and $\mathcal{B}[Y]$ are $\mathcal{B}$-free in $\left(\mathcal{A}, E_{\mathcal{B}}, \mathcal{B}\right)$. Then

$$
\Phi_{\mathcal{B}}^{*}(X, Y: \mathcal{B})=\Phi_{\mathcal{B}}^{*}(X: \mathcal{B})+\Phi_{\mathcal{B}}^{*}(Y: \mathcal{B}) .
$$

Proposition 2.8. [7] Let $\mathcal{B}, \mathcal{C}$ be von Neumann subalgebras of $\mathcal{A}$ satisfying $\mathcal{B} \subseteq$ $\mathcal{C}$ and $E_{\mathcal{B}}, E_{\mathcal{C}}$ be the conditional expectation of $\mathcal{A}$ onto $\mathcal{B}, \mathcal{C}$ respectively. Suppose $X=X^{*} \in \mathcal{A}$ satisfying $E_{\mathcal{B}[X]} E_{\mathcal{B}}=E_{\mathcal{B}}$, where $E_{\mathcal{B}[X]}: \mathcal{A} \rightarrow \mathcal{B}[X]$ is a conditional expectation. Then $\Phi_{\mathcal{B}}^{*}(X: \mathcal{B})=\Phi_{\mathcal{B}}^{*}(X: \mathcal{C}) \Longleftrightarrow X$ and $\mathcal{C}$ are $\mathcal{B}$-free.

The following two propositions are the generalizations of D. Voiculescu's corresponding results ([16]) in the operator-valued case.

Proposition 2.9. [7] (Analogue of Cramer-Rao inequality) $X_{1}, X_{2}, \cdots, X_{n} \in \mathcal{A}$ are self-adjoint, then the following equality holds:

$$
\Phi_{\mathcal{B}}^{*}\left(X_{1}, \cdots, X_{n}: \mathcal{C}\right) \sum_{i=1}^{n}\left\|E_{\mathcal{B}}\left(X_{i}^{*} X_{i}\right)\right\| \geq n^{2} I .
$$

Proposition 2.10. Let $1 \in \mathcal{B} \subseteq \mathcal{A}$ be a $*$-subalgebra. $X_{j}=X_{j}^{*} \in \mathcal{A}, X_{j}^{(k)}=$ $X_{j}^{(k) *} \in \mathcal{A}, 1 \leq j \leq n, k \in \mathbb{N}$ such that

(1) $X_{j}^{(k)} \longrightarrow X_{j}(k \rightarrow \infty)$ and the limit is in the sense of $\mathcal{B}$-distribution, i.e.

$\left\|E_{\mathcal{B}}\left(P\left(X_{j}^{(k)}\right)\right)-E_{\mathcal{B}}\left(P\left(X_{j}\right)\right)\right\| \longrightarrow 0(k \rightarrow \infty), \forall P$ is a polynomial over $\mathcal{B} ;$

$(2)\left\|\mathcal{J}_{\mathcal{B}}\left(X^{(k)}: \mathcal{B}\right)\right\|_{2} \leq C$, where $C$ is a constant and $\|x\|_{2}^{2}:=\left\|E_{\mathcal{B}}\left(x x^{*}\right)\right\|$. Then

$$
\liminf _{k \rightarrow \infty}\left\|\Phi_{\mathcal{B}}^{*}\left(X^{(k)}: \mathcal{B}\right)\right\| \geq\left\|\Phi_{\mathcal{B}}^{*}(X: \mathcal{B})\right\| .
$$

Proof. From the definition of conjugate variable, we have:

$$
\begin{aligned}
E_{\mathcal{B}} & \left(\mathcal{J}_{\mathcal{B}}(X: \mathcal{B}) b_{0} X b_{1} X \cdots X b_{n}\right) \\
& =\sum_{i} E_{\mathcal{B}}\left(b_{0} X \cdots X b_{j-1}\right) E_{\mathcal{B}}\left(b_{j} X \cdots X b_{n}\right) \\
& =\lim _{k \rightarrow \infty} \sum_{j=1}^{n} E_{\mathcal{B}}\left(\left(b_{0} X^{(k)} \cdots b_{j-1}\right)\right)\left(E_{\mathcal{B}}\left(b_{j} X^{(k)} \cdots b_{n}\right)\right) \\
& =\lim _{k \rightarrow \infty}\left(\mathcal{J}_{\mathcal{B}}\left(X^{(k)}: \mathcal{B}\right) b_{0} X^{(k)} b_{1} X^{(k)} \cdots b_{n}\right) \\
& =\lim _{k \rightarrow \infty} E_{\mathcal{B}}\left(\mathcal{J}_{\mathcal{B}}\left(X^{(k)}: \mathcal{B}\right) b_{0} X b_{1} X \cdots b_{n}\right) \\
& =\lim _{k \rightarrow \infty} E_{\mathcal{B}}\left(E_{\mathcal{B}[X]} \mathcal{J}_{\mathcal{B}}\left(X^{(k)}: \mathcal{B}\right) b_{0} X \cdots X b_{n}\right) .
\end{aligned}
$$

So we infer that

$$
\liminf _{k \rightarrow \infty}\left\|\Phi_{\mathcal{B}}^{*}\left(X^{(k)}: \mathcal{B}\right)\right\| \geq\left\|\Phi_{\mathcal{B}}^{*}(X: \mathcal{B})\right\| .
$$


Recall that a self-adjoint variable $X \in\left(\mathcal{A}, E_{\mathcal{B}}, \mathcal{B}\right)$ is called $\eta$-semicircular if it satisfies:

$$
\begin{cases}k_{\mathcal{B}}^{(1)}(X)=0 ; & \forall b \in \mathcal{B} ; \\ k_{\mathcal{B}}^{(2)}(X \otimes b X)=\eta(b), & \forall b_{1}, \cdots, b_{b} \in \mathcal{B}, n \geq 2 \\ k_{\mathcal{B}}^{(n+1)}\left(X \otimes b_{1} X \otimes \cdots \otimes b_{n} X\right)=0,\end{cases}
$$

where $\eta: \mathcal{B} \rightarrow \mathcal{B}$ is linear and $\left(k_{\mathcal{B}}^{(n)}\right)$ is the cumulant function induced by $E_{\mathcal{B}}$ (see [13]). For a semicircular variable with conditional expectation covariance (that is to say $\eta$ is a conditional expectation) its conjugate variable can be expressed by modular frames (see [8]). Frame is a notion that appears frequently in wavelets theory and it is a generalization of orthonormal basis in Hilbert space. D. Larson and some other people studied it from the view of operator theory. In [4, 5], M. Frank and D. Larson extended this notion to the Hilbert $C^{*}$-module framework and they have proved that most results of the Hilbert space frame can be generalized to the Hilbert $C^{*}$-module case. In this paper we mainly use some relations between the frames and the dual frames.

Definition 2.11. [4] Suppose $\left\{f_{i}\right\}_{i \in I} \subseteq L_{\mathcal{D}}^{2}(\mathcal{A})$ and satisfies: $\exists C, D>0$ such that

$$
C\langle x, x\rangle_{\mathcal{D}} \leq \sum_{i}\left\langle x, f_{i}\right\rangle_{\mathcal{D}}\left\langle f_{i}, x\right\rangle_{\mathcal{D}} \leq D\langle x, x\rangle_{\mathcal{D}}
$$

Then $\left\{f_{i}\right\}_{i \in I}$ will be called a modular frame in $L_{\mathcal{D}}^{2}(\mathcal{A})$. In particular, if $C=D=1$ then it will be called normalized tight. When the series in (2) converges in norm it will be called standard.

Note that in this paper we assume all of frames are standard.

Noting that a frame is a $\mathcal{D}$-linear dense set in the Hilbert $C^{*}$-module, in fact, we have:

Lemma 2.12. [4] (1) If $L_{\mathcal{D}}^{2}(\mathcal{A})$ is finitely or countably generated Hilbert $\mathcal{D}$ module, then it possesses a normalized tight frame.

(2) If $\left\{x_{i}\right\}$ is a frame in $L_{\mathcal{D}}^{2}(\mathcal{A})$, then there is a adjointable $\mathcal{D}$-linear operator $S$ : $L_{\mathcal{D}}^{2}(\mathcal{A}) \longrightarrow L_{\mathcal{D}}^{2}(\mathcal{A})$ (we call it frame operator and it is positive and invertible) such that $x=\sum_{j} S^{-1}\left(x_{j}\right)\left\langle x_{j}, x\right\rangle, \forall x \in \mathcal{L}_{\mathcal{D}}^{2}(\mathcal{A})$. In particular, when $\left\{x_{j}\right\}$ is normalized tight we have $x=\sum_{j} x_{j}<x_{j}, x>$.

If $\left\{y_{i}\right\}$ is a frame such that $x=\sum_{i} y_{i}<x_{i}, x>_{\mathcal{D}}$ then it will be called a dual frame of $\left\{x_{i}\right\}$.

In [8], we have computed out the free Fisher information of a semicircular variable with conditional expectation covariance by modular frames. Here we review some results.

Proposition 2.13. [8] Let $\mathcal{D} \subseteq \mathcal{B} \subseteq \mathcal{A}$ be a von Neumann subalgebras inclusion. $E: \mathcal{B} \rightarrow \mathcal{D}, E_{\mathcal{B}}: \mathcal{A} \rightarrow \mathcal{B}$ are conditional expectations and suppose $E_{\mathcal{D}}=E E_{\mathcal{B}}, L_{\mathcal{D}}^{2}(\mathcal{B})=\mathcal{B}$. Let $\left\{f_{i}\right\} \in L_{\mathcal{D}}^{2}(\mathcal{B})$ be a frame and suppose $S$ is the frame 
operator of $\left\{f_{i}\right\}$. Let $X=X^{*} \in\left(\mathcal{A}, E_{\mathcal{B}}, \mathcal{B}\right)$ be a E-semicircular variable. Then

$$
\begin{aligned}
& \mathcal{J}_{\mathcal{D}}(X: \mathcal{B})=\sum_{i} g_{i} X E\left(f_{i}^{*}\right) \\
& \mathcal{J}_{\mathcal{D}}(X: \mathcal{D})=X \\
& \mathcal{J}_{\mathcal{B}}(X: \mathcal{B})=\sum_{i} g_{i} X f_{i}^{*},
\end{aligned}
$$

where $g_{i}=S^{-1}\left(f_{i}\right), \forall i \in I$.

COROllary 2.14. [8] With the above notations and conditions. We have

$$
\begin{aligned}
& \Phi_{\mathcal{D}}^{*}(X: \mathcal{B})=\sum_{j} E\left(f_{j}\right) E\left(g_{j}^{*}\right)=1 ; \\
& \Phi_{\mathcal{D}}^{*}(X: \mathcal{D})=1 ; \\
& \Phi_{\mathcal{B}}^{*}(X: \mathcal{B})=\sum_{i} f_{i} g_{i}^{*} .
\end{aligned}
$$

Obviously, $\Phi_{\mathcal{B}}^{*}(X: \mathcal{B})=\sum_{i} f_{i} g_{i}^{*}=\operatorname{Ind}(E)($ see $[8])$.

3. Operator-valued relative free entropy. By the notion of relative free Fisher information and its properties, we can define the relative free entropy correspondly, prove some basic properties and show it is very closely related to the index of conditional expectations.

In [2], A. Connes and E. Størmer extended the notion of entropy to the nonabelian framework of operator algebras. The first step was to define the entropy of a finite dimensional subalgebra and more generally the relative entropy between two finite dimensional subalgebras $B_{1}, B_{2} \subseteq M$, as a substitute of the entropy and relative entropy for partitions: Let $M$ be a finite algebra with trace $\tau$ and let $S$ be the set of all finite families $\left(x_{1}, x_{2}, \cdots, x_{n}\right)$ of positive elements in $M$ with $\sum x_{i}=1$. If $\eta:[0, \infty) \rightarrow(-\infty, \infty)$ is defined by $\eta(t)=-t$ lnt , then

$$
H\left(B_{1} \mid B_{2}\right)=\sup _{\left(x_{i}\right) \in S} \sum_{i}\left(\tau \eta E_{B_{2}}\left(x_{i}\right)-\tau \eta E_{B_{1}}\left(x_{i}\right)\right)
$$

is the entropy of $B_{1}$ relative to $B_{2}$. If $B_{2}=\mathbb{C}$, then $H\left(B_{1} \mid \mathbb{C}\right)$ is simply the entropy of $B_{1}$.

In the particular case when $M$ is commutative and $B_{1}, B_{2}$ are generated by some partitions of the unity $P_{1}, P_{2}, H\left(B_{1} \mid B_{2}\right)$ coincides with the classical relative entropy $h\left(P_{1} \mid P_{2}\right)$. Quite surprisingly the above entropy is closely related to the index of subfactor. In fact, S. Popa proved if $N^{\prime} \cap M=\mathbb{C}$, then $H(M \mid N)=\ln [M: N]$, where $N \subseteq M$ is a subfactors inclusion(see [10]).

Now we follow D. Voiculescu's idea of defining the relative free entropy to define the operator-valued free entropy and study the relation between index and entropy. We do not restrict ourself to the $I I_{1}$ subfactors case. In fact we can consider general von Neumann algebras and the index of conditional expectations.

The index of conditional expectations was investigated by Y. Watatani, S. Popa etc[11, 14]. A conditional expectation $E: \mathcal{M} \rightarrow \mathcal{N}$ is said to be of finite index if there exists a positive scalar $k>0$ such that the mapping $k E-i d_{\mathcal{M}}$ is positive. Denote by $\operatorname{Ind}_{w}(E)$ (called the probabilistic index in [11]) the infimum of all such $k$. 
If there is no such $k$, put $\operatorname{Ind}_{w}(E)=\infty$. The condition $\operatorname{Ind}_{w}(E)<\infty$ is equivalent to the existence of a $\mathcal{N}$-orthonormal basis $\left\{m_{i}\right\}_{i \in I}$ of the $\mathcal{N}$-right Hilbert module $\mathcal{M}$ such that $\sum_{i} m_{i} m_{i}^{*}$ is weakly convergent. Moreover this limit is the same for every $\mathcal{N}$-orthonormal basis and is denoted by $\operatorname{Ind}(E)([1,11])$.

Before introducing the notion of operator-valued relative free entropy we introduce the integral in noncommutative von Neumann algebras first.

Let $\mathcal{B}$ be a von Neumann algebra on the Hilbert space $H$ and let $A(t) \in \mathcal{B}, t \in \mathbb{R}$. we say:

$$
\int_{0}^{\infty} A(t) d t=A
$$

that means:

$$
\int_{0}^{\infty}\langle A(t)(x), y\rangle d t=\langle A x, y\rangle
$$

for all $x, y \in H$. Obviously, $A \in \mathcal{B}$.

We mention some calculational properties of the above integral which we will use in this paper.

LEMMA 3.1. (1) $\int_{a}^{b} A \cdot A(t) d t=A \cdot \int_{a}^{b} A(t) d t$;

(2) $\int_{a}^{b}(A(t)-A) d t=\int_{a}^{b} A(t) d t-A(b-a)$; $-\ln A$.

(3) if $A$ is a positive and invertible operator, then $\int_{0}^{\infty} \frac{1}{1+t} I-A(I+t A)^{-1} d t=$

Proof. (1), (2) can be obtained directly by the definition and we only prove (3).

Since $A$ is positive and invertible, $\sigma(A) \in(0, \infty)$. Denote the spectral projections of $A$ by $E(z)$. we have:

$$
\begin{aligned}
\int_{0}^{\infty} & <\left(\frac{1}{1+t} I-A(1+t A)^{-1}\right)(x), y>d t \\
= & \int_{0}^{\infty} \int_{0}^{\infty}\left(\frac{1}{1+t}-z(1+t z)^{-1}\right) d\langle E(z)(x), y\rangle d t \\
= & \int_{0}^{\infty} \int_{0}^{\infty}\left(\frac{1}{1+t}-z(1+t z)^{-1}\right) d t d\langle E(z)(x), y\rangle \\
= & \left.\int_{0}^{\infty}(\ln (1+t)-\ln (1+t z))\right|_{0} ^{\infty} d\langle E(z)(x), y\rangle \\
= & \int_{0}^{\infty} \ln \frac{1}{z} d\langle E(z)(x), y\rangle=\langle-\ln A(x), y\rangle .
\end{aligned}
$$

Thus we obtain the result.

We introduce the notion of operator-valued relative free entropy as follows:

DeFinition 3.2. $\left(\mathcal{A}, E_{\mathcal{B}}, \mathcal{B}\right)$ is a $\mathcal{B}$-valued noncommutative probability space, and $\mathcal{B} \subseteq \mathcal{C} \subseteq \mathcal{A}$ is a subalgebra of $\mathcal{A}$. We define the $\mathcal{B}$-valued relative free entropy of self-adjoint variables $X_{1}, X_{2}, \cdots, X_{n}$ with respect to $\mathcal{C}$ by

$$
\begin{aligned}
& \chi_{\mathcal{B}}^{*}\left(X_{1}, X_{2}, \cdots, X_{n}: \mathcal{C}\right) \\
& \quad=\frac{1}{2} \int_{0}^{\infty}\left(\frac{n}{1+t} I-\Phi_{\mathcal{B}}^{*}\left(X_{1}+t^{\frac{1}{2}} S_{1}, \cdots, X_{n}+t^{\frac{1}{2}} S_{n}: \mathcal{C}\right)\right) d t+\frac{n}{2} \log 2 \pi e,
\end{aligned}
$$


where the $S_{j}^{\prime}$ s are $I$-semicircular and $\mathcal{C}\left[X_{1}, \cdots, X_{n}\right],\left\{S_{1}\right\}, \cdots,\left\{S_{n}\right\}$ are $\mathcal{B}$-free.

Proposition 3.3.

(1)

$$
\begin{aligned}
& \chi_{\mathcal{B}}^{*}\left(X_{1}, \cdots, X_{n}, Y_{1}, \cdots, Y_{m}: \mathcal{C}\right) \\
& \quad \leq \chi_{\mathcal{B}}^{*}\left(X_{1}, \cdots, X_{n}: \mathcal{C}\right)+\chi_{\mathcal{B}}^{*}\left(Y_{1}, \cdot Y_{m}: \mathcal{C}\right)
\end{aligned}
$$

(2) If $\mathcal{B}\left[X_{1}, \cdots, X_{n}\right]$ and $\mathcal{C}\left[Y_{1}, \cdots, Y_{m}\right]$ are $\mathcal{B}$-free, then

$$
\begin{aligned}
& \chi_{\mathcal{B}}^{*}\left(X_{1}, \cdots, X_{n}, Y_{1}, \cdots, Y_{m}: \mathcal{C}\right) \\
& \quad=\chi_{\mathcal{B}}^{*}\left(X_{1}, \cdots, X_{n}: \mathcal{B}\right)+\chi_{\mathcal{B}}^{*}\left(Y_{1}, \cdots, Y_{m}: \mathcal{C}\right)
\end{aligned}
$$

(3) If $\mathcal{B}\left[X_{1}, \cdots, X_{n}\right]$ and $\mathcal{C}$ are $\mathcal{B}-$ free, then

$$
\chi_{\mathcal{B}}^{*}\left(X_{1}, \cdots, X_{n}: \mathcal{B}\right)=\chi_{\mathcal{B}}^{*}\left(X_{1}, \cdots, X_{n}: \mathcal{C}\right) .
$$

Proof. All these are consequences of corresponding properties of $\Phi_{\mathcal{B}}^{*}$.

Proposition 3.4.

$$
\chi_{\mathcal{B}}^{*}\left(X_{1}, \cdots, X_{n}: \mathcal{C}\right) \leq-\frac{n}{2} \ln n+\frac{n}{2} \ln \left(\sum\left\|E_{\mathcal{B}}\left(X_{i}^{*} X_{i}\right)\right\|\right)+\frac{n}{2} \log 2 \pi e .
$$

Proof. By Proposition 2.9, we have:

$$
\begin{aligned}
& \Phi_{\mathcal{B}}^{*}\left(X_{1}+t^{\frac{1}{2}} S_{1}, \cdots, X_{n}+t^{\frac{1}{2}} S_{n}: \mathcal{C}\right) \sum_{i=1}^{n}\left\|E_{\mathcal{B}}\left(X_{i}+t^{\frac{1}{2}} S_{i}\right)^{*}\left(X_{i}+t^{\frac{1}{2}} S_{i}\right)\right\| \\
& \quad=\Phi_{\mathcal{B}}^{*}\left(X_{1}+t^{\frac{1}{2}} S_{1}, \cdots, X_{n}+t^{\frac{1}{2}} S_{n}: \mathcal{C}\right) \sum_{i=1}^{n}\left\|E_{\mathcal{B}}\left(X_{i}^{*} X_{i}\right)+t\right\| \\
& \quad \geq n^{2} I .
\end{aligned}
$$

It follows that

$$
\begin{aligned}
\chi_{\mathcal{B}}^{*} & \left(X_{1}, \cdots, X_{n}: \mathcal{C}\right) \\
& =\frac{1}{2} \int_{0}^{\infty}\left(\frac{n}{1+t} I-\Phi_{\mathcal{B}}^{*}\left(X_{1}+t^{\frac{1}{2}} S_{1}, \cdots, X_{n}+t^{\frac{1}{2}} S_{n}: \mathcal{C}\right)\right) d t+\frac{n}{2} \log 2 \pi e \\
& \leq \frac{1}{2} \int_{0}^{\infty}\left(\frac{n}{1+t} I-\frac{n^{2} I}{\sum\left\|E_{\mathcal{B}}\left(X_{i}^{*} X_{i}\right)\right\|+n t}\right) d t+\frac{n}{2} \log 2 \pi e \\
& =\frac{1}{2}\left(\left.n \ln (1+t)\right|_{0} ^{\infty}-\left.n \ln \left(\sum\left\|E_{\mathcal{B}}\left(X_{i}^{*} X_{i}\right)\right\|+n t\right)\right|_{0} ^{\infty}\right)+\frac{n}{2} \log 2 \pi e \\
& =-\frac{1}{2} \ln n+\frac{1}{2} n \ln \left(\sum\left\|E_{\mathcal{B}}\left(X_{i}^{*} X_{i}\right)\right\|\right)+\frac{n}{2} \log 2 \pi e .
\end{aligned}
$$

The following theorem is one of our main results in this paper.

Theorem 3.5. Let $E: \mathcal{B} \longrightarrow \mathcal{D}$ be a conditional expectation of $\mathcal{B}$ onto $\mathcal{D}$ of finite index and let $X=X^{*} \in \mathcal{A}$ is a $E$-semicircular variable. Then

$$
\chi_{\mathcal{B}}^{*}(X: \mathcal{B})=-\frac{1}{2} \ln (\operatorname{Ind}(E))+\frac{1}{2} \log 2 \pi e .
$$


To prove the above theorem we need several lemmas.

Lemma 3.6. [18] Let $X_{1}$ be $\eta_{1}$-semicircular, $X_{2}$ be $\eta_{2}$-semicircular and suppose $X_{1}, X_{2}$ are $\mathcal{B}$-free. Then $X_{1}+X_{2}$ is $\eta_{1}+\eta_{2}$-semicircular.

From the proof of Proposition 2.13 (see [8]), we have

Lemma 3.7. Let $\left(\mathcal{A}, E_{\mathcal{B}}, \mathcal{B}\right)$ be a noncommutative probability space and let $\mathcal{D} \subseteq \mathcal{B}$ be a subalgebra. If $\eta: \mathcal{B} \longrightarrow \mathcal{B}$ be a linear map satisfying $\eta\left(d_{1} b d_{2}\right)=d_{1} \eta(b) d_{2}$, $\forall d_{1}, d_{2} \in \mathcal{D}, b \in \mathcal{B}$ and there exist $\left\{f_{i}\right\},\left\{h_{i}\right\} \in \mathcal{B}$ such that $x=\sum_{i} h_{i} \eta\left(f_{i}^{*} x\right)=$ $\sum_{i} f_{i} \eta\left(h_{i}^{*} x\right), \forall x \in \mathcal{B}$, then for any selfadjoint $\eta$-semicircular variable $X \in \mathcal{A}$, we have $\stackrel{\mathcal{J}}{\mathcal{B}}(X: \mathcal{B})=\sum_{i} f_{i} X h_{i}^{*}$.

Lemma 3.8. Let $X=X^{*}$ be E-semicircular. Then for all $t \in \mathbb{R}$,

$$
\mathcal{J}_{\mathcal{B}}\left(X+t^{\frac{1}{2}} S: \mathcal{B}\right)=\sum_{i}(I+t \operatorname{Ind}(E))^{-1} f_{i}\left(X+t^{\frac{1}{2}} S\right) f_{i}^{*},
$$

where $\left\{f_{i}\right\}$ is a normalized tight modular frame in the inner product module $\left(\mathcal{B},\langle,\rangle_{\mathcal{D}}\right)$, where $\langle,\rangle_{\mathcal{D}}$ is the inner product induced by $E$.

Proof. It is easy to see $E+t I: \mathcal{B} \longrightarrow \mathcal{B}$ satisfying $(E+t I)\left(d_{1} b d_{2}\right)=d_{1}(E+$ $t I)(b) d_{2}, \forall b \in \mathcal{B}, d_{1}, d_{2} \in \mathcal{D}$ and for all $x \in \mathcal{B}$,

$$
\begin{aligned}
\sum_{i} & (I+t \operatorname{Ind}(E))^{-1} f_{i}(E+t I)\left(f_{i}^{*} x\right) \\
& =(I+\operatorname{Ind}(E))^{-1}\left(x+\sum_{i} t f_{i} f_{i}^{*} x\right) \\
& =(I+t \operatorname{Ind}(E))^{-1}(x+t \operatorname{Ind}(E) x) \\
& =(I+t \operatorname{Ind}(E))^{-1}(1+t \operatorname{Ind}(E)) x \\
& =x .
\end{aligned}
$$

Hence from lemma 3.7 we obtain the desired result.

Proof of Theorem 3.5.

$$
\begin{aligned}
& \Phi_{\mathcal{B}}^{*}\left(X+t^{\frac{1}{2}} S: \mathcal{B}\right) \\
& \quad=\sum_{i, j} E_{\mathcal{B}}(I+t \operatorname{Ind} E)^{-1} f_{i}\left(X+t^{\frac{1}{2}} S\right) f_{i}^{*} \\
& f_{j}\left(X+t^{\frac{1}{2}} S\right)\left(\left((I+t \operatorname{Ind} E)^{-1} f_{j}\right)^{*}\right) \\
& \quad=\sum_{i, j}(I+t \operatorname{IndE})^{-1} f_{i}(E+t I)\left(f_{i}^{*} f_{j}\right) f_{j}^{*}(I+t \operatorname{Ind} E)^{-1} \\
& \quad=\sum_{j} f_{j} f_{j}^{*}(I+t \operatorname{Ind} E)^{-1} .
\end{aligned}
$$


Hence

$$
\begin{aligned}
\chi_{\mathcal{B}}^{*}(X: \mathcal{B}) & =\frac{1}{2} \int_{0}^{\infty}\left(\frac{1}{1+t} I-\Phi_{\mathcal{B}}^{*}\left(X+t^{\frac{1}{2}} S: \mathcal{B}\right)\right) d t+\frac{1}{2} \log 2 \pi e \\
& =\frac{1}{2} \int_{0}^{\infty}\left(\frac{1}{1+t} I-\sum_{j} f_{j} f_{j}^{*}(I+t \operatorname{Ind} E)^{-1}\right) d t+\frac{1}{2} \log 2 \pi e \\
& =\frac{1}{2} \int_{0}^{\infty}\left(\frac{1}{1+t} I-\operatorname{IndE}(I+t \operatorname{IndE})^{-1}\right) d t+\frac{1}{2} \log 2 \pi e \\
& =\left.\frac{1}{2} \ln (1+t)(1+t \operatorname{IndE})^{-1}\right|_{0} ^{\infty}+\frac{1}{2} \log 2 \pi e \\
& =\frac{1}{2} \ln \ln d^{-1} E+\frac{1}{2} \log 2 \pi e \\
& =-\frac{1}{2} \ln (\operatorname{IndE})+\frac{1}{2} \log 2 \pi e .
\end{aligned}
$$

Note that $\ln (\operatorname{IndE})$ is in the sense of functional calculus.

Corollary 3.9. If $\mathcal{D} \subseteq \mathcal{B} \subseteq \mathcal{A}$ are $I I_{1}$ factors inclusion and $\mathcal{D}^{\prime} \cap \mathcal{B}=\mathbb{C}$. $E: \mathcal{B} \rightarrow \mathcal{D}$ is the trace-preserving conditional expectation and let $X=X^{*}$ be a E-semicircular variable then

$$
\chi_{\mathcal{B}}^{*}(X: \mathcal{B})=-\frac{1}{2} H(\mathcal{B} \mid \mathcal{D}) I+\frac{1}{2} \log 2 \pi e .
$$

4. An estimate for free entropy dimension. According to D. Voiculescu's work, we can define the free (relative) entropy dimension for random variables. The estimate of entropy dimension is very important since it has been applied to solve many classical problems in operator algebras such as D. Voiculescu's and Ge's famous work (See [15],[6]). Now our definition of free (relative) entropy dimension is different from the notion in [16] since we defined it in terms of the operator-valued free relative entropy.

Definition 4.1. Let $X_{1}, X_{2}, \cdots, X_{n} \in\left(\mathcal{A}, E_{\mathcal{B}}, \mathcal{B}\right)$, and let $\tau$ be a state on $\mathcal{A}$. Define the free entropy dimension $\delta_{\mathcal{B}, \tau}^{*}$ of $X_{1}, \cdots, X_{n}$ w.r.t. $\mathcal{B}, \tau$ by

$$
\delta_{\mathcal{B}, \tau}^{*}\left(X_{1}, \cdots, X_{n}\right)=n+\limsup _{\varepsilon \rightarrow 0} \tau\left(\frac{\chi_{\mathcal{B}}^{*}\left(X_{1}+\sqrt{\varepsilon} S_{1}, \cdots, X_{n}+\sqrt{\varepsilon} S_{n}\right)}{|\ln \sqrt{\varepsilon}|}\right)
$$

where $S_{i}^{\prime}$ s are $I$-semicircular and $\left\{S_{1}\right\}, \cdots,\left\{S_{n}\right\},\left\{X_{1}, \cdots, X_{n}\right\}$ are $\mathcal{B}$-free.

Before estimate $\delta_{\mathcal{B}, \tau}^{*}$, we need do some preparations.

Lemma 4.2. Let $X, Y \in\left(\mathcal{A}, E_{\mathcal{B}}, \mathcal{B}\right)$ be $\mathcal{B}$-free and let $\mathcal{B} \subseteq \mathcal{C} \subseteq \mathcal{A}$. Let $\mathcal{J}_{\mathcal{B}}(X: \mathcal{C})$ exist and let $E_{\mathcal{C}} E_{\mathcal{C}[X+Y]}=E_{\mathcal{C}}$. Then $\mathcal{J}_{\mathcal{B}}(X+Y: \mathcal{C})$ exists and $\mathcal{J}_{\mathcal{B}}(X+Y: \mathcal{C})=$ $E_{\mathcal{C}[X+Y]} \mathcal{J}_{\mathcal{B}}(X: \mathcal{C})$.

Proof. We use Lemma 2.5 to prove this lemma and the notations be as the same as in Lemma 2.5. By the definition of conjugate variables and Theorem 2.5 in [9] we 
have

$$
\begin{aligned}
& k_{\mathcal{C}}^{(1)}\left(E_{\mathcal{C}[X+Y]} \mathcal{J}_{\mathcal{B}}(X: \mathcal{C})\right)=E_{\mathcal{C}}\left(\mathcal{J}_{\mathcal{B}}(X: \mathcal{C})\right)=0 \\
& k_{\mathcal{C}}^{(2)}\left(E_{\mathcal{C}[X+Y]} \mathcal{J}_{\mathcal{B}}(X: \mathcal{C}) \otimes c(X+Y)\right) \\
& \quad=k_{\mathcal{C}}^{(2)}\left(E_{\mathcal{C}[X+Y]} \mathcal{J}_{\mathcal{C}}(X: \mathcal{C}) \otimes c X\right)+k_{\mathcal{C}}^{(2)}\left(E_{\mathcal{C}[X+Y]} \mathcal{J}_{\mathcal{C}}(X: \mathcal{C}) \otimes c Y\right) \\
& \quad=k_{\mathcal{C}}^{(2)}\left(E_{\mathcal{C}[X+Y]} \mathcal{J}_{\mathcal{C}}(X: \mathcal{C}) \otimes c X\right) \\
& \quad=E_{\mathcal{B}}(c) ; \\
& k_{\mathcal{C}}^{m+1}\left(E_{\mathcal{C}[X+Y]} \mathcal{J}_{\mathcal{B}}(X: \mathcal{C}) \otimes c_{1}(X+Y) \otimes \cdots \otimes c_{m}(X+Y)\right)=0
\end{aligned}
$$

where $m \geq 1$ and $c, c_{1}, \cdots, c_{m} \in \mathcal{C}$.

Lemma 4.3. Let $X_{1}, X_{2}, \cdots, X_{n} \in \mathcal{A} .\left\{S_{1}\right\}, \cdots,\left\{S_{n}\right\}$ are E-semicircular and $\mathcal{B}$-free from $\left\{X_{1}, \cdots, X_{n}\right\}$. Then

$$
\Phi_{\mathcal{B}}^{*}\left(X_{1}+\sqrt{\varepsilon} S_{1}, \cdots, X_{n}+\sqrt{\varepsilon} S_{n}: \mathcal{B}\right) \leq \Phi_{\mathcal{B}}^{*}\left(X_{1}, \cdots, X_{n}: \mathcal{B}\right) .
$$

Proof. From the definition

$$
\begin{aligned}
& \Phi_{\mathcal{B}}^{*}\left(X_{1}+\sqrt{\varepsilon} S_{1}, \cdots, X_{n}+\sqrt{\varepsilon} S_{n}: \mathcal{B}\right) \\
& \quad=\sum_{i=1}^{n} E_{\mathcal{B}}\left(\mathcal{J}_{\mathcal{B}}\left(X_{i}+\sqrt{\varepsilon} S_{i}: \mathcal{B}\left[X_{1}+\sqrt{\varepsilon} S_{1}, \cdots, \widehat{X_{i}+\sqrt{\varepsilon}} S_{i}, \cdots, X_{n}+\sqrt{\varepsilon} S_{n}\right]\right) ;\right. \\
& \left(\mathcal{J}_{\mathcal{B}}\left(X_{i}+\sqrt{\varepsilon} S_{i}: \mathcal{B}\left[X_{1}+\sqrt{\varepsilon}, \cdots, X_{i}+\widehat{\sqrt{\varepsilon}} S_{i}, \cdots, X_{n}+\sqrt{\varepsilon} S_{n}\right]\right)^{*}\right. \\
& \quad \leq \sum_{i=1}^{n} E_{\mathcal{B}}\left(E_{\mathcal{B}\left[X_{1}+\sqrt{\varepsilon} S_{1}, \cdots, X_{n}+\sqrt{\varepsilon} S_{n}\right]} \mathcal{J}_{\mathcal{B}}\left(X_{i}: \mathcal{B}\left[X_{1}, \cdots, \widehat{X_{i}}, \cdots, X_{n}\right]\right) ;\right. \\
& \left.E_{\mathcal{B}\left[X_{1}+\sqrt{\varepsilon} S_{1}, \cdots, X_{n}+\sqrt{\varepsilon} S_{n}\right]} \mathcal{J}_{\mathcal{B}}\left(X_{i}: \mathcal{B}\left[X_{1}, \cdots, \widehat{X_{i}}, \cdots, X_{n}\right]\right)^{*}\right) \\
& \quad \leq \sum_{i=1}^{n} E_{\mathcal{B}}\left(\mathcal{J}_{\mathcal{B}}\left(X_{i}: \mathcal{B}\left[X_{1}, \cdots, \widehat{X_{i}}, \cdots, X_{n}\right]\right) \mathcal{J}_{\mathcal{B}}\left(X_{i}: \mathcal{B}\left[X_{1}, \cdots, \widehat{X_{i}}, \cdots, X_{n}\right]\right)^{*}\right) \\
& \quad=\Phi_{\mathcal{B}}^{*}\left(X_{1}, \cdots, X_{n}: \mathcal{B}\right),
\end{aligned}
$$

where $E_{\mathcal{B}\left[X_{1}+\sqrt{\varepsilon} S_{1}, \cdots, X_{n}+\sqrt{\varepsilon} S_{n}\right]}$ is the conditional expectation of $\mathcal{A}$ onto $\mathcal{B}\left[X_{1}+\right.$ $\left.\sqrt{\varepsilon} S_{1}, \cdots, X_{n}+\sqrt{\varepsilon} S_{n}\right]$ such that $E_{\mathcal{B}} E_{\mathcal{B}\left[X_{1}+\sqrt{\varepsilon} S_{1}, \cdots, X_{n}+\sqrt{\varepsilon} S_{n}\right]}=E_{\mathcal{B}}$. The first inequality follows from Proposition 2.6 and Lemma 4.2 and the second follows from the conditional expectation's Cauchy-Schwartz inequality.

Lemma 4.4. With the above notations, we have

$$
\Phi_{\mathcal{B}}^{*}\left(X_{1}+\sqrt{\varepsilon} S_{1}, \cdots, X_{n}+\sqrt{\varepsilon} S_{n}: \mathcal{B}\right) \leq \frac{n}{\varepsilon} \operatorname{IndE} .
$$


Proof.

$$
\begin{aligned}
\Phi_{\mathcal{B}}^{*} & \left.X_{1}+\sqrt{\varepsilon} S_{1}, \cdots, X_{n}+\sqrt{\varepsilon} S_{n}: \mathcal{B}\right) \\
= & \sum_{i=1}^{n} E_{\mathcal{B}}\left(\mathcal{J}_{\mathcal{B}}\left(X_{i}+\sqrt{\varepsilon} S_{i}: \mathcal{B}\left[X_{1}+\sqrt{\varepsilon} S_{1}, \cdots, \widehat{X_{i}+\sqrt{\varepsilon}} S_{i}, \cdots, X_{n}+\sqrt{\varepsilon} S_{n}\right]\right)\right. \\
& \cdot\left(\mathcal{J}_{\mathcal{B}}\left(X_{i}+\sqrt{\varepsilon} S_{i}: \mathcal{B}\left[X_{1}+\sqrt{\varepsilon}, \cdots, \widehat{X_{i}+\sqrt{\varepsilon}} S_{i}, \cdots, X_{n}+\sqrt{\varepsilon} S_{n}\right]\right)^{*}\right. \\
\leq & \sum_{i=1}^{n} E_{\mathcal{B}}\left(\left(E _ { \mathcal { B } [ X _ { 1 } + \sqrt { \varepsilon } S _ { 1 } , \cdots , X _ { n } + \sqrt { \varepsilon } S _ { n } ] } \left(\mathcal{J}_{\mathcal{B}}\left(\sqrt{\varepsilon} S_{i}: \mathcal{B}\left(\sqrt{\varepsilon} S_{1}, \cdots, \widehat{\sqrt{\varepsilon} S_{i}}, \cdots, \sqrt{\varepsilon} S_{n}\right)\right)\right.\right.\right. \\
& \left.\cdot\left(E_{\mathcal{B}\left[X_{1}+\sqrt{\varepsilon} S_{1}, \cdots, X_{n}+\sqrt{\varepsilon} S_{n}\right]}\left(\mathcal{J}_{\mathcal{B}}\left(\sqrt{\varepsilon} S_{i}: \mathcal{B}\left(\sqrt{\varepsilon} S_{1}, \cdots, \widehat{\sqrt{\varepsilon} S_{i}}, \cdots, \sqrt{\varepsilon} S_{n}\right)\right)\right)\right)^{*}\right) \\
\leq & \sum_{i=1}^{n} E_{\mathcal{B}}\left(E_{\mathcal{B}\left[X_{1}+\sqrt{\varepsilon} S_{1}, \cdots, X_{n}+\sqrt{\varepsilon} S_{n}\right]} \mathcal{J}_{\mathcal{B}}\left(\sqrt{\varepsilon} S_{i}: \mathcal{B}\right) \cdot E_{\mathcal{B}\left[X_{1}+\sqrt{\varepsilon} S_{1}, \cdots, X_{n}+\sqrt{\varepsilon} S_{n}\right]} \mathcal{J}_{\mathcal{B}}\left(\sqrt{\varepsilon} S_{i}: \mathcal{B}\right)^{*}\right) \\
\leq & \sum_{i=1}^{n} E_{\mathcal{B}}\left(\mathcal{J}_{\mathcal{B}}\left(\sqrt{\varepsilon} S_{i}: \mathcal{B}\right) \cdot \mathcal{J}_{\mathcal{B}}\left(\sqrt{\varepsilon} S_{i}: \mathcal{B}\right)^{*}\right) \\
= & \frac{n}{\varepsilon} \Phi_{\mathcal{B}}^{*}\left(S_{i}: \mathcal{B}\right) \\
= & \frac{n}{\varepsilon} \operatorname{Ind}(E) .
\end{aligned}
$$

The last equality follows from Corollary 2.14.

THEOREM 4.5. With the above notations, we have

$$
\delta_{\mathcal{B}, \tau}^{*}\left(X_{1}, \cdots, X_{n}: \mathcal{B}\right) \geq n .
$$

In addition if $X_{1}, \cdots, X_{n}$ is a $\mathcal{B}$-free family of I-semicircular variables in $\mathcal{A}$ then

$$
\delta_{\mathcal{B}, \tau}^{*}\left(X_{1}, \cdots, X_{n}: \mathcal{B}\right)=n .
$$

Proof. Let $\left\{S_{1}^{\prime}\right\}, \cdots,\left\{S_{n}^{\prime}\right\} \quad$ be $I$-semicircular and $\mathcal{B}$-free from $\left\{X_{1}, \cdots, X_{n} ; S_{1}, \cdots, S_{n}\right\}$ and let $0 \leq \varepsilon \leq 1, M=\Phi_{\mathcal{B}}^{*}\left(X_{1}, \cdots, X_{n}: \mathcal{B}\right)$.

$$
\begin{aligned}
\chi_{\mathcal{B}}^{*}( & \left.X_{1}+\sqrt{\varepsilon} S_{1}, \cdots, X_{n}+\sqrt{\varepsilon} S_{n}: \mathcal{B}\right) \\
= & \frac{1}{2} \int_{0}^{\infty}\left(\frac{n}{1+t}-\Phi_{\mathcal{B}}^{*}\left(X_{1}+\sqrt{\varepsilon} S_{1}+\sqrt{t} S_{1}^{\prime}, \cdots, X_{n}+\sqrt{\varepsilon} S_{n}+\sqrt{t} S_{n}^{\prime}: \mathcal{B}\right)\right) d t \\
& \quad+\frac{n}{2} \log 2 \pi e \\
= & \frac{1}{2} \int_{0}^{\infty} \frac{n}{1+t}-\Phi_{\mathcal{B}}^{*}\left(X_{1}+\sqrt{\varepsilon+t} S_{1}, \cdots, X_{n}+\sqrt{\varepsilon+t} S_{n}: \mathcal{B}\right) d t+\frac{n}{2} \log 2 \pi e \\
= & \frac{1}{2} \int_{\varepsilon}^{\infty} \frac{n}{1+t-\varepsilon}-\Phi_{\mathcal{B}}^{*}\left(X_{1}+\sqrt{t} S_{1}, \cdots, X_{n}+\sqrt{t} S_{2}\right) d t+\frac{n}{2} \log 2 \pi e \\
\geq & \frac{1}{2} \int_{\varepsilon}^{1} \frac{n}{1+t-\varepsilon}-\Phi_{\mathcal{B}}^{*}\left(X_{1}, \cdots, X_{n}: \mathcal{B}\right) d t+\frac{1}{2} \int_{1}^{\infty}\left(\frac{n}{1+t-\varepsilon}-\frac{n}{t}\right) d t+\frac{n}{2} \log 2 \pi e \\
= & \frac{1}{2}\left(\left.n \ln (1+t-\varepsilon)\right|_{\varepsilon} ^{1}-M(1-\varepsilon)+n \ln (2-\varepsilon)\right) I+\frac{n}{2} \log 2 \pi e \\
= & \frac{1}{2}(n \ln (2-\varepsilon)-M(1-\varepsilon)+n \ln (2-\varepsilon)) I+\frac{n}{2} \log 2 \pi e
\end{aligned}
$$


The second equality holds because the distribution of $\sqrt{\varepsilon} S_{n}+\sqrt{t} S_{n}^{\prime}$ is the same as that of $\sqrt{\varepsilon+t} S_{n}$ by Lemma 3.6.

So from the definition of $\delta_{\mathcal{B}, \tau}^{*}$,

$$
\delta_{\mathcal{B}, \tau}^{*}\left(X_{1}, \cdots, X_{n}: \mathcal{B}\right) \geq n .
$$

When $X_{1}, \cdots, X_{n}$ is a $\mathcal{B}$-free family of $I$-semicircular variables, we have:

$$
\begin{aligned}
\chi_{\mathcal{B}}^{*}( & \left.X_{1}+\sqrt{\varepsilon} S_{1}, \cdots, X_{n}+\sqrt{\varepsilon} S_{n}: \mathcal{B}\right) \\
= & \frac{1}{2} \int_{0}^{\infty}\left(\frac{n}{1+t} I-\Phi_{\mathcal{B}}^{*}\left(X_{1}+\sqrt{\varepsilon} S_{1}+\sqrt{t} S_{1}^{\prime}, \cdots, X_{n}+\sqrt{\varepsilon} S_{n}+\sqrt{t} S_{n}^{\prime}: \mathcal{B}\right)\right) d t \\
& +\frac{n}{2} \log 2 \pi e I \\
= & \frac{1}{2} \int_{0}^{\infty}\left(\frac{n}{1+t} I-\Phi_{\mathcal{B}}^{*}\left(X_{1}+\sqrt{\varepsilon+t} S_{1}, \cdots, X_{n}+\sqrt{\varepsilon+t} S_{n}: \mathcal{B}\right)\right) d t+\frac{n}{2} \log 2 \pi e I \\
= & \frac{1}{2} \int_{\varepsilon}^{\infty}\left(\frac{n}{1+t-\varepsilon} I-\Phi_{\mathcal{B}}^{*}\left(X_{1}+\sqrt{t} S_{1}, \cdots, X_{n}+\sqrt{t} S_{2}\right)\right) d t+\frac{n}{2} \log 2 \pi e I
\end{aligned}
$$

where $X_{1}, \cdots, X_{n} ; S_{1}, \cdots, S_{n} ; S_{1}^{\prime}, \cdots, S_{n}^{\prime}$ is a free family with amalgamation over $\mathcal{B}$ and $S_{1}, \cdots, S_{n} ; S_{1}^{\prime}, \cdots, S_{n}^{\prime}$ are all $I$-semicircular variables.

And since $X_{i}+\sqrt{t} S_{1}$ are $(1+t) I$-semicircular variables,

$$
\Phi_{\mathcal{B}}^{*}\left(X_{1}+\sqrt{t} S_{1}, \cdots, X_{n}+\sqrt{t} S_{n}\right)=\frac{n}{1+t} I .
$$

Thus $\delta_{\mathcal{B}, \tau}^{*}\left(X_{1}, \cdots, X_{n}\right)=n+\limsup _{\varepsilon \rightarrow 0} \tau\left(\frac{\chi_{\mathcal{B}}^{*}\left(X_{1}+\sqrt{\varepsilon} S_{1}, \cdots, X_{n}+\sqrt{\varepsilon} S_{n}\right)}{|\ln \sqrt{\varepsilon}|}\right)=n$.

\section{REFERENCES}

[1] M. Baillet, Y. Denizeau and J.-F. Havet, Indice d'une esperance conditionelle, Compositio Math., 66 (1998), pp. 199-236.

[2] A. Connes et E. Störmer, Entropy for automorphisms of $I I_{1}$ von Neumann algebras, Acta Math, 134 (1975), pp. 288-306.

[3] T. M. Cover and J. A. Thomas, Elements of information theory, John Wiley \& Sons, Inc. Chichester(1976).

[4] M. Frank, D. R. Larson, A module frame concept for Hilbert $C^{*}{ }_{-}$modules, in: Functional and Harmonic Analysis of Wavelets (San Antonio, TX, Jan. 1999), A.M.S., Providence, R.I., Contemp. Math., 247 (2000), pp. 207-233.

[5] M. Frank, D. Larson, Frames in Hilbert $C^{*}$-modules and $C^{*}$-algebras, J. Operator Theory, 48 (2002), pp. 273-314.

[6] L. Ge, Applications of free entropy to finite von neumann algebrasII, Annals of Mathematics, 147 (1998), pp. 143-157.

[7] B. Meng, M. Guo, X. CaO, Free Fisehr information and amalgamated freeness, Appl. Math. Mech., 25:10 (2004), pp. 1007-1013.

[8] B. Meng, M. Guo, X. CaO, Free Fisher information and modular frames, to appear in Proc. Amer. Math. Soc.

[9] A. Nica, D. Shlyakhtenko, R. Speicher, Operator-valued distributions. 1. Characterizations of freeness, IMRN 2002:29 (2002), pp. 1509-1538.

[10] M. Pimsner, S. Popa, Entropy and index for subfactors, Ann scient. Ec. Norm. Sup., 4:19 (1986), pp. 57-106.

[11] S. POPA, Clsssification of subfactors and their endomorphisms, CMBS 86, AMS (1995).

[12] R. SPEICHER, Combinatorial theory of the free product with amalgamation and operator-valued free probability theory, Memoirs of AMS No. 627 (1998), pp. 1-88.

[13] V. Sunder, An invitation to von Neumann algebras, Springer-Verlag (1987).

[14] Y. Watatani, Index for $C^{*}$-subalgebras, Memoirs AMS 83:424 (1990). 
[15] Dan Voiculescu, The analogues of entropy and of fisher's information measure in free probability theory III: The absence of cartan subalgebras, Geometric and Functional Analysis, 6:1 (1996), pp. 172-199.

[16] Dan Voiculescu, The analogues of entropy and of Fisher's information measure in free probability theory V: Noncommutative Hibert Transforms, Inventiones mathematicae, 132 (1998), pp. 189-227.

[17] Dan Voiculescu, The analogues of entropy and of fisher's information measure in free probability theory VI: Liberation and mutual free information, Advances in Mathematics, 146 (1999), pp. 101-166.

[18] Dan Voiculescu, Operations on certain Non-commutative operator-valued random variables, Astérisque, 232 (1995), pp. 243-275. 